\author{
Ahmet N. Kök \\ Omer S. Yildirim \\ Mehmet Kizilkaya \\ Ali F. Isik \\ Nasuhi E. Aydin \\ Talat Yurtman
}

\section{The detrimental effect of neck pain followed by solitary plasmacytoma resulting in spontaneous quadriplegia: a case report}

Received: 8 October 2003

Accepted in revised form: 1 March 2004

\begin{abstract}
A.N. Kök
Department of Forensic Medicine,

Faculty of Medicine, Ataturk University, Erzurum, Turkey
\end{abstract}

O.S. Yildirim ( $\square)$

Surgery Orthopaedic,

Faculty of Medicine, Ataturk University, Erzurum, Turkey

e-mail: omers@atauni.tr

Tel.: +90-442-316-6333 ext. 2127

Fax: +90-442-316-3340

\section{Kizilkaya}

Department of Anesthesia and Reanimation, Faculty of Medicine, Ataturk University, Erzurum, Turkey

\section{A.F. Isik \\ Department of Forensic Medicine, Faculty of Medicine, \\ Gazi University, Ankara, Turkey}

\section{N.E. Aydin}

Department of Pathology,

Faculty of Medicine,

Inonu University,

Malatya, Turkey

T. Yurtman

Council of Forensic Medicine,

Ankara, Turkey

\begin{abstract}
After complaining of neck pain for 3 weeks, a 36-year-old man suddenly become quadriplegic during a massage session in a traditional Turkish bath. He was immediately transferred to hospital, where the initial examination consisting in plain radiography and magnetic resonance imaging revealed a compression fracture of the fourth cervical vertebra. The patient was immediately treated by anterior vertebral decompression and bone grafting from the iliac crest. Unfortunately, the patient did not respond to surgical intervention and died. Solitary plasmacytoma of the fourth cervical vertebrae was detected at autopsy during forensic examination. Pain and spinal cord compression resulted from solitary plasmacytoma of the vertebral column. However, paralysis, especially quadriplegia, occurs seldom. In this case report, solitary plasmacytoma of the vertebral column associated with neck pain is presented with the relevant pathological and clinical aspects.
\end{abstract}

Key words Solitary plasmacytoma • Neck pain • Trivial injury • Quadriplegia 


\section{Introduction}

Plasmacytoma, also known as myeloma, is a neoplastic proliferation of plasma cells that may develop in different forms. About 5\%-25\% of patients with plasmacytoma have a solitary lesion revealing no evidence of dissemination [1, 2]. However, most of these lesions progress to develop multiple or generalised myeloma [2, 3]. Solitary plasmacytoma usually involves the spine, whereas multiple myeloma can localise to any bone that contains red marrow in adult life [2-4]. We discuss a fatal case of solitary plasmacytoma of the vertebral column with the relevant pathological and clinical aspects. The disease was observed in a 36-year-old patient who had become quadriplegic during a massage session after complaining of neck pain for 3 weeks. We emphasize the importance of knowledge on health status prior to massage therapy.

\section{Case report}

A 36-year-old single man working as a mining engineer had been complaining of unremitting pain in the neck region for a period of 3 weeks. Several physicians had examined him and prescribed analgesics along with application of warm or hot water to relieve the neck muscle spasm. In a traditional Turkish bath, he suddenly became quadriplegic during forceful massage on his neck. At the first examination in the hospital, the patient was imaged by plain radiography and magnetic resonance imaging (MRI) of the neck region (Fig 1). The MRI section revealed a compression-burst fracture in the fourth cervi-

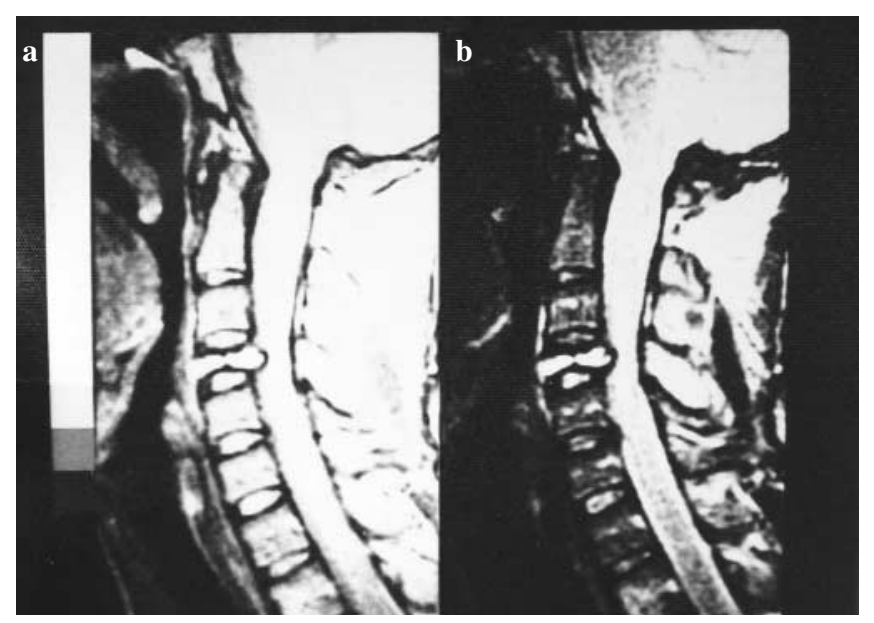

Fig. 1a, b Magnetic resonance images of the neck region showing fracture of the fourth cervical vertebrae (a) and cord compression with edema (b) on T1-weighted sagittal view

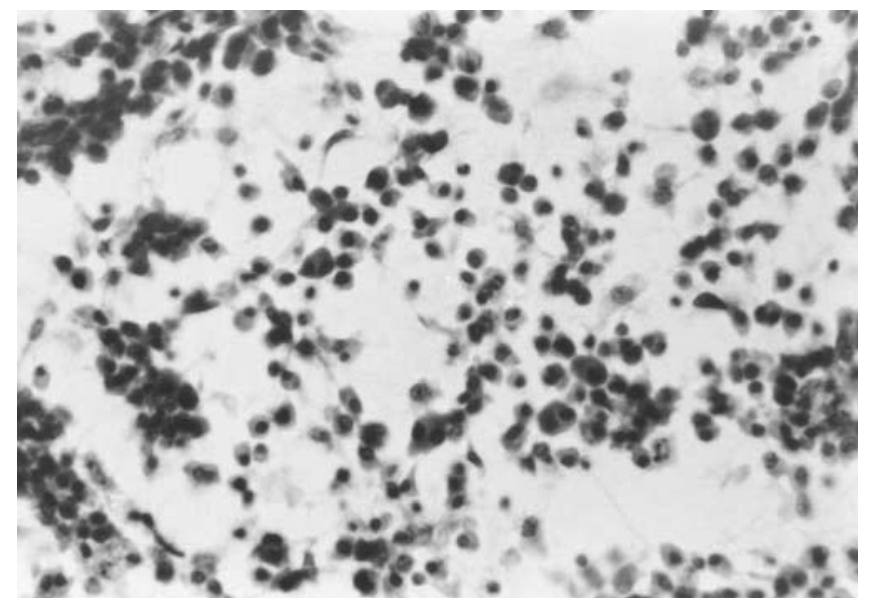

Fig. 2 Microscopic section revealing dense monotonous plasmacellular infiltration, proliferation of abnormal plasma cells and poor differentiation in focal areas (H\&E, x100)

cal vertebrae and edema in the medulla spinalis. Thus, at first, surgical decompression was attempted. In the surgical intervention, after anterior decompression, a strut graft obtained from the iliac crest was placed as described by Bohlman and Eismont [5]. After administration of a loading dose of $30 \mathrm{mg} / \mathrm{kg}$ prednisolone (Prednisolon Amp Fako; Istanbul, Turkey), the patient continued to receive antiedematous treatment with $5.4 \mathrm{mg} / \mathrm{kg}$ prednisolone for 48 hours. Following operation, he was transferred to the intensive care unit. He was conscious with motion of the diaphragm. The patient died one day after the surgical intervention.

To fulfill legal requirements, a complete autopsy was performed. Edematous brain, fragility of the fourth cervical vertebrae remnant along with hemorrhagic spinal cord, and bilaterally edematous lungs were detected in the internal examination. The microscopic section of the fourth cervical vertebrae remnant revealed a monotonous infiltration of plasma cells with binuclear and remarkably large forms (Fig. 2). The plasmacellular infiltration involved both the bone fragments and soft tissue parts in the vicinity with presence of no other focuses, assuring a case of solitary plasmacytoma.

\section{Discussion}

In this case, failure to detect the presence of a tumour and ignorance of neck pain at the earlier examination led to quadriplegia, which eventually led to the death of patient. The massage action was not an assault but a forceful motion, which probably fractured the weakened vertebrae as a result of a solitary plasmacytoma. Surgical treatment 
was intended to stabilise the spine, reduce mechanical compression, and decompress neurologic elements. Surgery was essential in this particular case because of urgency and possible unresponsiveness to radiotherapy. Tumour removal in the upper cervical spine using individual modified surgical strategies is based on tumour location, cervical stabilisation, and vertebral body replacement. These significantly minimize complications and increase life span and quality [6].

Physical therapy is one of the most commonly used treatments for mechanical neck disorders and requires a careful evaluation of a patient's health status [7]. Physical therapy incorporates a large variety of methods such as mobilizing exercises, stretching, isometric, static or dynamic strengthening, endurance training, and proprioceptive exercises. Success from physical therapy increases in patients with definite neurological defects excluding neck pain caused by diffuse connective tissue disease, rheumatic syndromes, metabolic and endocrine diseases, neurological disease, neoplasms, fractures, and dislocation [7, 8], suggesting that obtaining information about health status is crucial prior to application of physical therapy.

Cervical manipulation occasionally causes serious vertebrobasilar complications. The general cause is vertebral artery dissection. In some cases however there has been no obvious arterial injury [9]. The rarity of vertebrobasilar complications makes this association difficult to study despite high volumes of chiropractic treatment. Because of the popularity of spinal manipulation, high-quality research on both its risks and benefits is recommended.
Plasmacytomas occurs predominantly in males; in particular, the solitary osseous form ocurs in men of about 50 years of age [1-3]. The solitary plasmacytoma mainly localises in the spine $[2-4,10]$. The most common clinical symptom is pain due to bone involvement [1-3]. Bone destruction may occur as a result of an uncoupling process associated with stimulation of osteoclastic resorption as well as an inhibition of bone formation [10]. Because of plasmacytoma localised in the cervical spine, the patient had been suffering from neck pain. This patient was much younger than patients described in other case reports [1-3]. The clinical presentation was characterized by pathological fracture of the fourth cervical vertebrae after a trivial trauma during the massage session in a Turkish bath. In this patient, the neck pain obviously had been ignored in his previous examinations. Quadriplegia was treated by anterior vertebral decompression and bone grafting from the iliac crest. However, the patient could not recover and died one day after surgery. Clinical observations and autopsy findings revealed that cause for the death was not related to respiratory insufficiency in regard to injury of the phrenic nerve. However, presence of edema hinted that death was possibly related to the secondary involvement of the vital structures at the brain stem.

In summary, this report presented a unique case which may help physicans manage patients with early stages of cervical vertebral tumours associated with neck pain in order to eliminate undesirable consequences. Premanipulative testing of the cervical spine and complete examination covering nerve function, bone structure, and body motion are crucial for success of rehabiltation programme for neck pain.

\section{References}

1. Dimopoulos MA, Moulopoulos A, Delasalle K, Alexanian R (1992) Solitary plasmacytoma of bone and asymptomatic multiple myeloma. Hematol Oncol Clin North Am 6:359-369

2. Mirra J (ed) (1989) Bone tumours. Lea Febiger, Philadelphia London, pp 1121-1141

3. Campanacci M (ed) (1990) Bone and soft tissue tumours. Springer, Berlin Heidelberg New York, pp 559-574
4. Bacci G, Savini R, Calderoni P et al (1982) Solitary plasmacytoma of the vertebral column. A report of 15 cases. Tumori 68:271-275

5. Bohlman HH, Eismont FJ (1981) Surgical techniques of anterior decompression and fusion for spinal cord injuries. Clin Orthop 154:57-67

6. Vieweg U, Rao G, Stoffel J, Meyer B (2000) Tumor surgery of the upper cervical spine. Chirurg 71(9):1144-1151

7. Hing WA, Reid DA, Monaghan M (2003) Manuplation of the cervical spine. Man Ther 8(1):2-9
8. Clubb D (2002) Cervical manipulation and vertebral artery injury: a literature review. J Man Manipulative Ther 10(1):11-16

9. Mann T, Refshauge KM (2001) Causes of complications from cervical spine manipulation. Aust J Physiother 47(4):255-266

10. Bataille R, Chappard D, Klein B (1992) Mechanisms of bone lesions in multiple myeloma. Hematol Oncol Clin North Am 6:285-295 